\title{
A Review of Recent Wake Vortex Research for Increasing Airport Capacity
}

\author{
James N. Hallock ${ }^{a}$ Frank Holzäpfel ${ }^{b *}$ \\ ${ }^{\text {a }}$ Retired, Waltham MA, USA \\ ${ }^{b}$ Institute of Atmospheric Physics, German Aerospace Center (DLR) Oberpfaffenhofen, \\ D-82234 Wessling, Germany
}

\begin{abstract}
This paper is a brief review of recent wake vortex research as it affects the operational problem of spacing aircraft to increase airport capacity and throughput. The paper addresses the questions of what do we know about wake vortices and what don't we know about wake vortices. The introduction of Heavy jets in the late 1960s stimulated the study of wake vortices for safety reasons and the use of pulsed lidars and the maturity of computational fluid dynamics in the last three decades have led to extensive data collection and analyses which are now resulting in the development and implementation of systems to safely decrease separations in the terminal environment. Although much has been learned about wake vortices and their behavior, there is still more to be learned about the phenomena of aircraft wake vortices.
\end{abstract}

Key Words: aircraft wake vortices; wake behaviour; meteorological effects; wake decay; lidar; computational fluid dynamics

\section{Introduction}

In January 1991, one of us (JNH) published a report [1] that proposed alternative strategies for the US wake vortex program based on the then current knowledge of wake vortices. The expansion of the US program and the addition of many international wake vortex programs inspired the preparation of this updated review of the situation. This paper used the 1991 report as a starting point; the material herein is an update bringing the reader to 2018 by addressing the questions:

\footnotetext{
*Corresponding author. Tel.: +49-8153-282529, fax: +49-8153-281841.

E-mail address: frank.holzaepfel@dlr.de (F. Holzäpfel)

Published in Progress in Aerospace Sciences, 2018, https://doi.org/10.1016/i.paerosci.2018.03.003
} 
1. What do we know about wake vortices?

2. What don't we know about wake vortices?

within the context of increasing airport capacity. Two major additions to the state of knowledge of aircraft wake vortices in the last three decades are the introduction of the pulsed lidar as a data collection device and the use of computational fluid dynamics (CFD) in describing the behavior of wake vortices. The emphasis of this paper will be the results of analyses of both experimental data and numerical simulations. However, extra effort was expended in this paper to address the historical development of CFD; such an overview has not been given before and the historical context helps to better understand the limitations and benefits of CFD. A measure of the current state of knowledge is the introduction of systems at airports to mitigate the conservative fixed separations between aircraft in the terminal environment (RECAT in the US and RECAT-EU in Europe).

Pulsed lidar can track vortices at altitudes up to 1500 feet (457 meters) and for long translational distances. The lidar processing algorithm identifies the two vortex centers and the velocity distributions and, by matching the velocity distributions to a model, yields the vortex strengths or circulations. Pulsed lidars have been used to monitor vortex behavior at various airports and locations along the final approach path and the initial takeoff path.

CFD has become a mature tool supporting the consistent investigation of wake vortex behavior under various environmental conditions and in ground proximity and even specific phenomena like the formation of double rings or vortex bursting have become tangible. However, CFD has not yet had a significant impact on aircraft spacing for airport capacity enhancement. This leads to the question what will be required to give safety regulators more confidence in CFD for safety purposes. The advantage of CFD is that almost all variables of interest are readily available for analysis. Although benchmarks between different simulation codes feature satisfactory agreement for various scenarios, substantial differences for vortex behavior in, for example, turbulent environments remain depending on the characteristics of the adopted turbulence. First examples of consistent consideration of the aircraft type, configuration and flight phase are emerging but need further development and validation to become a means for reliable assessments of specific scenarios.

The wake vortex problem is complex because of the large number of variables. Setting aside the various operational scenarios, the problem involves the parameters introduced by the vortexgenerating aircraft, by the vortex-encountering aircraft and by the intervening atmosphere. The vortex is initially characterized by the parameters of the vortex-generating aircraft (weight, wingspan, speed, flap and spoiler settings, proximity to the ground, engine thrust, lift distribution, etc.). The encounter (safe or hazardous) is characterized by the parameters of the 
following aircraft (speed, wingspan, roll control authority, phase of flight, etc.). The meteorology (wind with its components headwind and crosswind, wind shear, atmospheric stability, turbulence, etc.) plays a leading role in determining how long a vortex remains hazardous.

Many surveys and reviews on wake vortex research have been published over the years. In 1975 the Annual Review by Widnall [2] and the extensive monograph of Donaldson and Bilanin [3] appeared, the latter still being a repository for analytical wake vortex methods. Hallock and Eberle [4] edited a state-of-the-art review of the US wake vortex R\&D program as input for the ICAO ninth Air Navigation Conference held in 1977 to address wake vortex effects and separation standards. Twenty-one years later, Hallock et al. [5] provided a retrospection on mainly the US wake vortex activities and Spalart [6] presented his discerning and sobering review on the understanding of wake vortex physics as relevant to safety and productivity of aviation. One year later in 1999, Rossow [7] gave a historical review with a focus on wake structure and alleviation. In the year 2002, Gerz et al. [8] presented a consolidated European view on the status of knowledge on aircraft wake characteristics, technical and operational procedures of minimizing and predicting vortex strength, and avoiding wake encounters. Further research needs reports compiled within WakeNet-Europe networks appeared in the following years [9],[10]. Breitsamter [11] gave a brief overview on past and present wake vortex research, followed by detailed reports on wind tunnel investigations including turbulence and instability characteristics.

\section{Current Knowledge of Vortex Behavior}

Any finite lifting wing must leave behind it two counter-rotating trailing vortices, the direction of rotation being such that between these vortices the air moves downwards while outside of them the induced flow is upwards. The wake vortex originates in the vorticity shed from the generating wing (see Fig. 1). The vorticity can be resolved into streamwise (oriented with the flight direction) and cross-stream (aligned perpendicular to the flight path). If the wing contains significant regions of concentrated streamwise or cross-stream perturbations (due to control surfaces, flaps, spoilers, landing gear, etc.), there may be more than one vortex pair, and various stages may develop with different time scales compared to the clean-wing case. The various vortices interact and eventually combine into a single pair. The different stages may be delayed or accelerated; this situation occurs for aircraft in the landing or takeoff configurations.

Aerodynamics dominates the rollup process, but the ambient atmosphere eventually dictates how the vortices behave. Vortex motion and decay are stochastic processes; i.e., the vagaries of the atmosphere and slight changes in aircraft characteristics can lead to different vortex 
behavior even though it seems that all the conditions are the same. Stochastic processes require extensive data collection to determine the envelope of behavior.

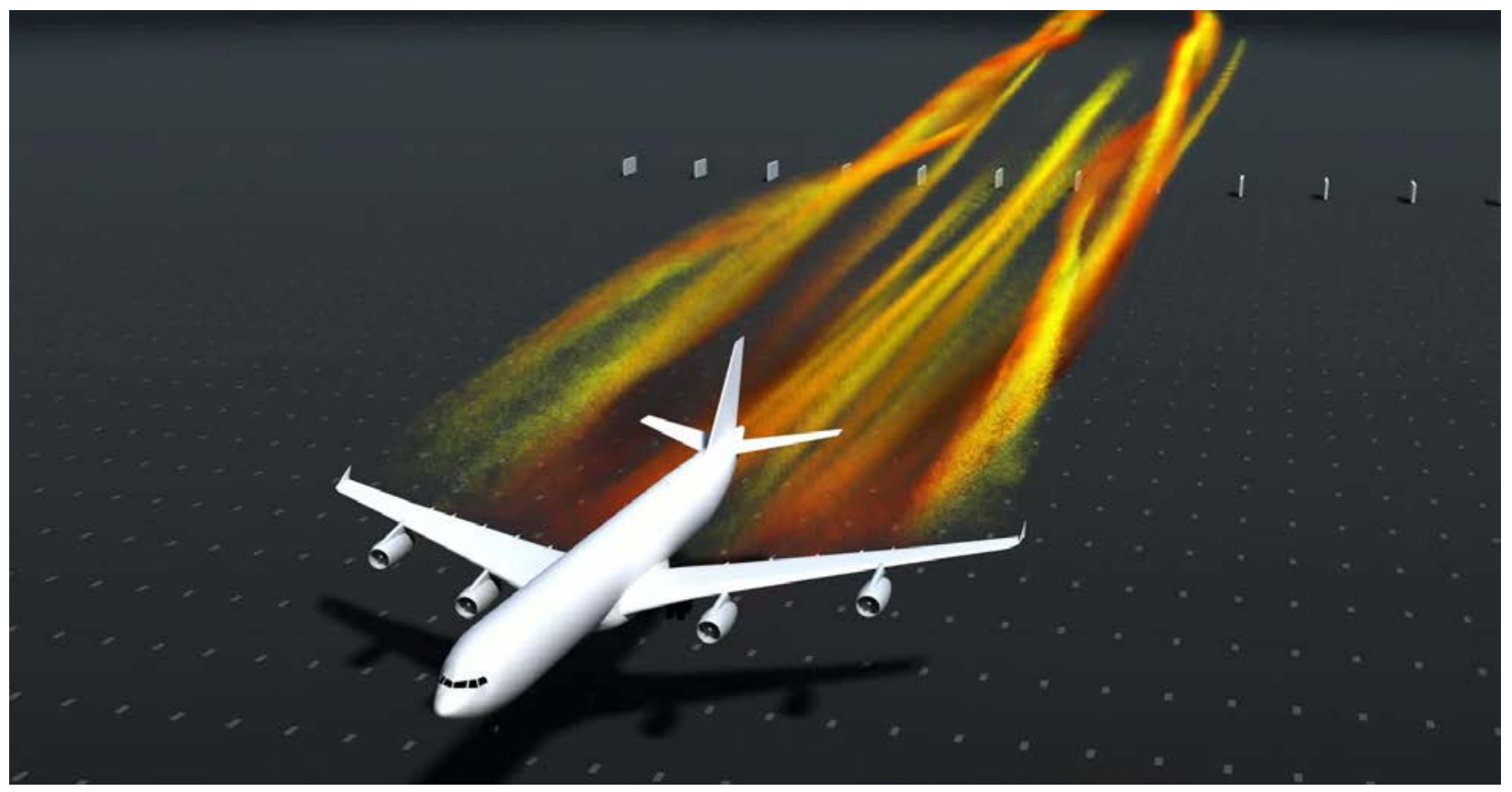

Fig. 1. Wake-vortex rollup (visualized by vorticity distribution) during final approach of aircraft in high-lift configuration [12].

\subsection{Motion near the Ground}

The primary mechanism of vortex transport is mutual induction, that is, vortex motion is caused by each vortex being in the velocity field of the other vortex. Ground effect is calculated using image vortices, which are imaginary vortices whose presence creates the same effect as the ground plane, thereby obviating the need to otherwise model the ground plane. In the absence of wind shear, the vortices are of equal strength and descend together. It has been observed that vortices tend to descend to an altitude of about one-half of their initial separation (see Fig. 2). In inviscid flow and without crosswind, the vortex trajectory is a hyperbola. However, the upwind vortex can be expected to stall over the runway if the crosswind is approximately equal to the initial descent speed.

Extensive measurements indicate that the vortex pair upon reaching the point of closest approach to the ground will then rise in altitude. This is known as rebound and comes about due to the generation of a weak secondary pair of vortices outside and below the vortex pair as it nears the ground. The separation of the two vortices in ground effect leads to the familiar situation where a crosswind equal to half the speed of the vortex separation could cause the upwind vortex to stall over the runway. Lidar measurements, numerical simulations, and 
encounter analyses indicate that this most critical crosswind magnitude roughly corresponds to the initial vortex descent speed.

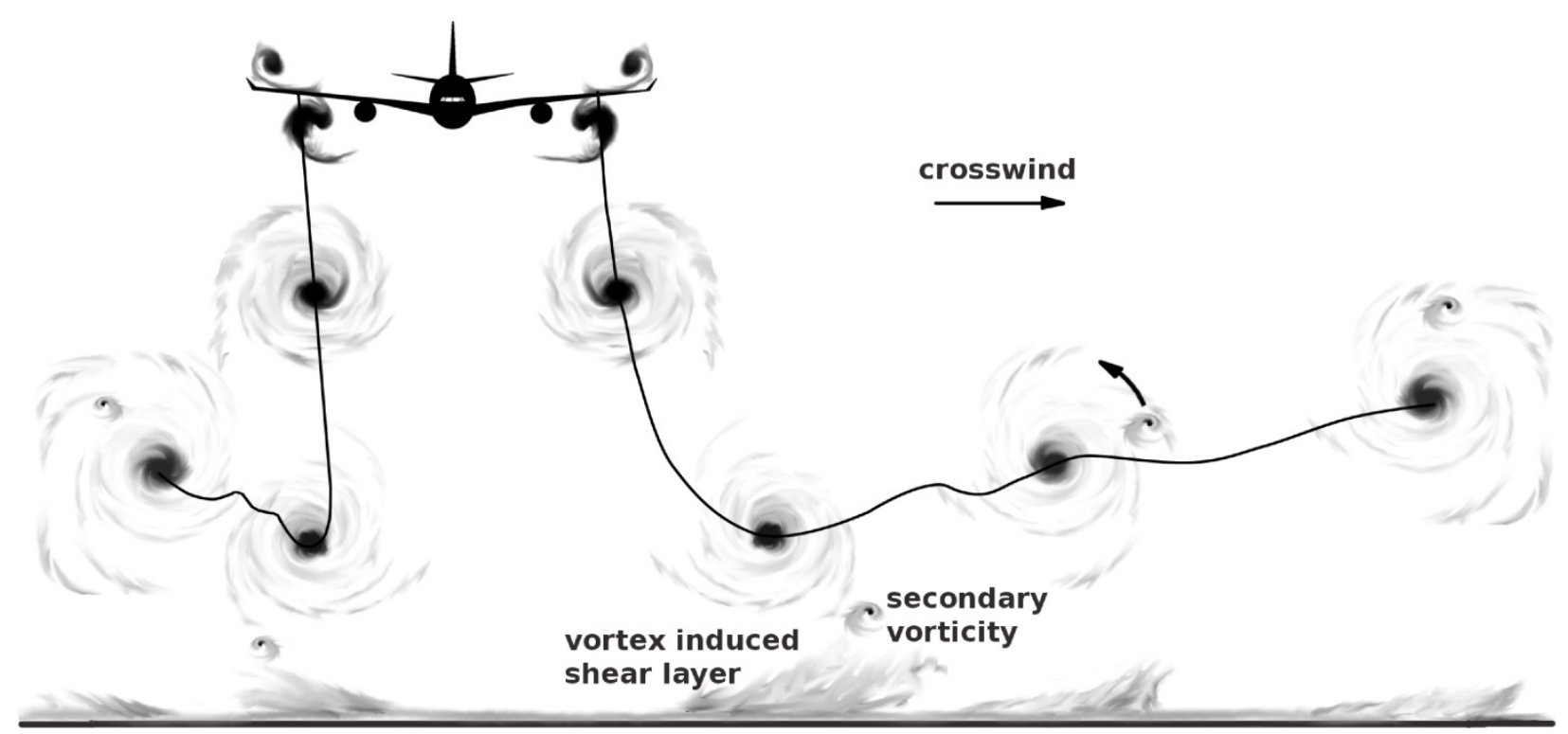

boundary layer

Fig.2. Vortex evolution in ground proximity [13]. Vorticity distributions from numerical simulations [14] and trajectories from fast-time prediction model [15].

Crosswind shear modifies the trajectories of vortices near the ground by causing unequal decay of the vortex pair. The unequal decay leads to unsymmetrical vortex motion where typically the downwind vortex will rise and the upwind vortex will sink lower in altitude thus exhibiting vortex tilt.

\subsection{Velocity Flow Field}

Early research centered on measuring the tangential velocity profiles and the core radius of wake vortices using propeller or hot-wire anemometers mounted on towers [16]. Various aircraft were flown upwind of the tower. Often smoke canisters were also mounted on the tower to mark the location of the two vortices where they hit the tower as well as their subsequent motion downwind of the tower. From these measurements, the vortex circulation was extracted by fitting the tower data to various vortex models.

Many of the new vortex sensors were tested along with the tower measurements as the smoke indicated the vortex locations in time and the tower measurements gave the initial conditions of vortex location, vortex characteristics and the attendant wind field [17]. Such vortex sensors included propeller anemometers (windlines), ultraviolet, infrared, acoustic (pulsed - bistatic and monostatic) and lidar (continuous wave and pulsed). Although the velocity flow field was 
of interest, it soon was replaced by the vortex location and the vortex circulation (derived from the flow field and core size) as a function of time.

A literature research comprising laboratory investigations, numerical simulations and flight tests indicates that no universal tangential velocity profile can be identified. Instead, all known velocity profiles described in [8] are found with a certain accumulation around the HallockBurnham vortex. A numerical simulation [18] reveals that directly behind the wing Jacquin's multiple scale vortex model may be found transforming into the Lamb-Oseen vortex model during roll-up and finally approaching the Hallock-Burnham vortex after roll-up. Thus, literature indicates that there is no universal tangential velocity profile. Instead tangential velocity profiles, on one hand, appear to depend on the Reynolds (Re) number; on the other hand, highRe vortices undergo a development where the interplay between turbulence, which is strongly damped in the centrifugally stable vortex core region, and rotation strives for some equilibrium.

\subsection{Lateral Motion}

Vortices move with the wind and near the ground the motion is affected by the presence of the ground [19]. The lateral motion of vortices is important in the airport environment as a vortex could translate from one runway area into another runway region. Approximately 50 years ago the maximum lateral motion of a vortex from one runway region to another was measured and was used as input to the "2500-foot" (762 meters) rule whereby operations on parallel runways are considered to be independent in VFR from a wake vortex perspective if the runways are separated by at least 2500 feet (762 meters). Subsequently, it has been shown that vortices from many aircraft move laterally 750 feet or 229 meters (San Francisco International Airport [21]) and even 1700 feet or 518 meters (Frankfurt International Airport [20]) between parallel runways, but the downwind vortices decay faster than the upwind vortices.

\subsection{Vertical Motion}

The initial descent rate of the wake is adequately described by classical theory; the descent rate is determined by the weight, flight speed and wingspan of the generating aircraft. Generally, vortices descend at the initial rate of about 300 to 500 feet per minute (91 to 152 meters per minute) for about 30 seconds [22]. The descent rate then decreases and eventually approaches zero at between 500 and 900 feet (152 and 274 meters) below the flight path for most Medium commercial aircraft. However, the vortices from the very heavy aircraft (A-380, B-747-400, B747-8, etc.) have been observed at and below 1000 feet (305 meters) below their flight path at en route altitudes.

In thermally stably stratified environments the oval around the vortex pair descends and warms adiabatically. The evolving buoyancy force reduces the descent speed and in strongly stably stratified environments may cause vortex stall or even rebound. While under cruise conditions 
the stable stratification close to the tropopause region usually limits vortex descent, rather rare weakly stable conditions enable descent of vortices shed by Heavy aircraft to and even beyond the adjacent flight level $1000 \mathrm{ft}$ below [23].

\subsection{Decay Processes}

Many decades of flow visualization, whether from on-board or tower-mounted smoke sources, show that, for all large aircraft, after vortex roll up, the flow in the vortex core always appears laminar with no turbulence. Even the complex roll-up processes that generate rotorcraft wake vortices result in laminar cores [24]. The laminarization of the core flow may be related to the centrifugal stability in the core that inhibits turbulent fluctuations. For laminar cores the only direct dissipation mechanism is viscosity, which leads to very slow decay. The effective demise of a vortex thus might be related more to distortion (e.g., Crow instability [25]).

The region outside the vortex core contains turbulence that comes from two sources: the aircraft and the atmosphere. Direct measurements of this turbulence have been made with tower-mounted hot-wire anemometers. Turbulence can cause the observed decay of vorticity in the outer vortex region via turbulent diffusion. When this vorticity is gone, the circulation profile becomes flat for aged vortices [26].

The Rayleigh instability criterion dictates that the vortex circulation must at least remain constant for neutral stability or increase with radius for stable conditions. Therefore, countersign vorticity wrapped around the outside of a vortex can effectively annihilate the outer vorticity of the vortex and force the circulation profile to be constant beyond a certain radius.

The decay process of the wake is complex and is strongly influenced by the atmospheric conditions. The decay process is driven by the following factors:

Atmospheric Turbulence. Atmospheric turbulence plays a significant role in the decay of the vortex. Large-scale atmospheric turbulence deforms the vortex whereas smallerscale atmospheric turbulence imparts viscous forces on the wake. These forces extract energy from the vortex, thus reducing its strength. The heavier the turbulence, the quicker the wake decays.

Viscous Interactions. The viscosity of the atmosphere slowly extracts energy from the vortex, thus reducing its strength at a very slow rate.

Buoyancy. An upward force acts on the vortex as a result of the density inside the vortex system being lower than the density outside the vortex. Counter-rotating baroclinic vorticity is produced along the vortex oval representing the interface between lower 
and higher density. Complex interactions of baroclinic vorticity and primary vorticity slowly extract energy from the vortex and thus reduce its strength [27].

Vortex Instability. A small amount of turbulence in the atmosphere can create instability in the vortex pair that causes the vortices to link. Crow showed that this instability comes from a combination of mutual interactions between the sinusoidally deformed vortices and self-induced motion. When the vortices link, the strength of the pair decays.

Secondary Vorticity Structures. Vortices deform and decay primarily from the overlapping of oppositely signed vorticity, caused by vorticity stretching and azimuthal structures. Secondary vorticity may originate from atmospheric turbulence, baroclinic vorticity or interaction with the ground surface. Stretching, tilting and merger of ambient vorticity by the primary vortices create coherent secondary vortices out of the ambient incoherent flow [28]. Counter-rotating vorticity streaks produced midway between the vortices can be effective in exchanging fluid across the symmetry line causing mutual annihilation of primary vorticity [27]. The creation and stretching of secondary vorticity structures consumes energy of the primary wake vortices [29].

Proximity to the ground (or the somewhat equivalent stratification of the atmosphere) is known to accelerate the rate of vortex decay. The mechanism for such increased decay is enhancement of the external countersign vorticity that detaches from the ground and wraps around the primary vortices. Likewise, countersign windshear vorticity promotes vortex decay, while same-sign windshear vorticity reduces vortex decay. Wake distortion by the Crow instability, driven by atmospheric turbulence, is often the dominant decay mechanism that mitigates the wake encounter hazard. Away from the ground the Crow instability results from the interaction of the wake vortex pair. Near the ground the two wake vortices separate and the Crow instability can take place with the image vortex (used to satisfy the boundary condition of zero vertical flow at the ground).

There are several schools of thought regarding how wake vortices decay reaching from continuous to three-phased decay characteristics. Whereas some lidar measurements indicate continuous circulation decay [30], others feature two-phase decay [31]. Hallock [32] measured similar behaviour using pulsed acoustic measurements. The different observed circulation decay characteristics of lidar data may at least partly be related to different data processing techniques.

Large eddy simulations (LES) feature nearly linear circulation decay in strongly turbulent environments [33],[34]. Two-phase decay is characterized by an initial phase of gradual decay termed "diffusion phase" followed by a "rapid decay phase" which typically commences shortly 
before the vortices link. This behaviour is observed for weak to moderate turbulence and in ground proximity. A third phase of decay is most prevalent for wakes imbedded within environments having low-turbulence and near-neutral stratification. The third decay phase, with a more gradual rate of decay, follows the formation of ring vortices.

Various studies as well as operational experience have identified the region near the ground on approach (nominally, one wingspan of the wake generator above the ground) as the worst-case region for a wake encounter. In this region, wakes will not descend below the glide slope and an encountering aircraft would have little altitude or time to apply power to recover from a wake encounter. Vortex data collections in the US, Germany, United Kingdom, France, Japan and the United Arab Republic have therefore included this region in their wake vortex research programs. Analyses of the data focused on the longest lasting vortices, typically, the top five percent of the longest lasting cases, which generally occurred in low winds with low turbulence. Ultimately, the result was a nondimensional decay curve with the circulation normalized by the initial circulation and the time normalized by the time for the vortex pair to descend a distance of one initial vortex separation.

\subsection{Vortex Alleviation}

Vortex alleviation is a general term used to describe modifications made to the airframe of the wake-generating aircraft for the purpose of decreasing the effect of trailing vortices on a following aircraft. The goal is to decrease the allowable longitudinal spacing between aircraft by modifying the vortex structure in such a way that if a vortex encounter does occur, the resulting upset will not exceed the control authority of the following aircraft. Alleviation is usually achieved by modification of the spanwise wing loading or by the generation of turbulence behind the wake-generating aircraft.

Many techniques have been tried in wind tunnels, water channels, numerical simulations and in flight tests. To date, nothing has been developed for use in approach or departure operations. Even from a requirements standpoint there are issues. For example, if the device increases drag of the vortex-generating aircraft, will an operator install such a device if it increases fuel burn and the benefit is only for the following aircraft?

Many aircraft are now equipped with winglets. The first production aircraft with winglets was the Lear Longhorn 28/29 in 1977. Winglets are designed to lessen drag when flying en route and thereby lessen the amount of fuel burned. There have been many claims that winglets also lessen the wake turbulence. During approach and landing, the primary vortex comes from the outboard edge of the outboard flap and not from the wingtip where the winglet is placed. Extensive measurements of wake vortex decay comparing approaching aircraft make-modelseries with and without winglets show no discernible differences [35]. 
The installation of so-called plate lines beyond the runway ends may accelerate wake vortex decay during final approach; the flight phase where most encounters are reported [12],[36]. A plate line consists of a series of individual plates aligned parallel to the runway direction. While descending the wake vortices interact with the plates generating disturbances that propagate along the vortices in and against the flight direction. Flight tests indicate that these disturbances may reduce the lifetime of the longest-lived and potentially most hazardous wake vortices by one-third [36].

\subsection{Computational Fluid Dynamics}

Early numerical studies in the 1960s employed the method of discrete vortex approximation in order to model, for example, the rolling up of a vortex sheet [37], a numerical method which was later used for fast-time models because of its numerical efficiency (see section 2.8). Later on, this method was also applied to simulate vortex evolution in a thermally stably stratified environment, where the generated baroclinic vorticity was represented by discrete vortices in order to model the decelerated descent of the vortex pair [38].

In the 1980s, two-dimensional (2D) unsteady numerical simulations employing, for example, finite differences and second order turbulence closures appeared. Impressive agreement of vortex descent and even circulation evolution on different radii with measurements of $B 747$ vortices [39] was achieved in stably stratified turbulent environments given appropriate adjustments of vortex core size and turbulent macroscale [40]. Subsequent CFD studies with refined codes and increased mesh resolution did not bring along automatically better agreement with observations. For example, the well-resolved baroclinic vorticity layer forming in stably stratified environments along the vortex oval led to excessive detrainment causing vortex cores to approach each other with the consequence of substantially accelerated vortex descent speeds [41].

Such erroneous findings could only be overcome by the extension of the codes to the third dimension enabling the formation of three-dimensional (3D) instability mechanisms. It was in the year 1996, when the first journal publication with 3D numerical simulations appeared [42]. Again, stratification effects were studied and Ref. [42] already resolved up to seven Crow wavelengths triggered by different perturbation waves. Obviously, in 1996 when Ref. [43] saw not less than seven groups presenting their first 3D simulations, the time was ripe for these computationally much more expensive approaches. While Spalart and Wray [44] studied the impact of atmospheric turbulence on the formation of the Crow instability, Gerz and Ehret [45] focused on the wake dynamics and the exhaust distribution behind cruising aircraft. Corjon et al. [28] already resolved and interpreted the formation of secondary vorticity structures generated from environmental turbulence in the strain field of the wake vortices (see section 2.5). 
Further refinement of computational methods and power were yielding more and more realistic results [46],[47]. The resolution of 3D decay mechanisms was decisive to prevent, for example, the unrealistic accelerated descent in stably stratified environments observed in 2D [27]. Pre-runs of the atmospheric boundary layer or stably stratified turbulence allowed for the development of realistic environments to which the counter-rotating velocity field of the wake vortices was superimposed. That way even specific phenomena like the formation of double rings or vortex bursting became tangible [48].

Still most numerical simulations initialize the wake vortices employing analytical vortex models like the Lamb-Oseen or Burnham-Hallock vortex (see [8]), yielding a so-called temporal simulation, where the peculiarities of vortex roll-up are not considered and the vortex age does not vary along the flight direction. Ref. [49] did a first step towards real 3D by visualizing temporal simulations as so-called drift plots yielding a fully 3D representation reaching from the initialization of Gaussian vorticity distributions until final decay. Ref. [50] went a further step by conducting steady 3D Euler simulations of the flow around the aircraft and continuing these in a temporal simulation of the roll-up process from a plane extracted downstream of the fuselage end.

The first fully 3D simulations also termed spatial simulations starting from the flow around the aircraft over the subsequent vortex roll-up until final decay were conducted by [14]. For this purpose, the flow field surrounding an aircraft obtained from Reynolds-averaged Navier-Stokes simulation was flown as an internal boundary condition through the LES domain to initialize the wake. This method was later extended to simulate the final approach of an aircraft in high-lift configuration including touchdown on the runway [12]. This way the propagation of so-called end effects appearing after touchdown and weakening the vortices during this critical phase of flight could be analyzed.

One way to assess the accuracy and reliability of numerical simulations of aircraft wake vortices is comparing CFD results with field observations. Ref. [51] reproduced the spectacular rebound of an upwind vortex above a group of trees at London Heathrow Airport employing a relaxation scheme to model the complex variable wind field. Ref. [52] demonstrated that the formation of a solitary vortex with unusual long lifetime can be related to the first and second vertical derivatives of the ambient crosswind. These two examples demonstrate that even exceptional vortex behavior can be well reproduced by CFD given sufficiently accurate initial and boundary conditions and modeling effort.

Alternatively, a benchmark of different CFD codes may be instructive. The unstable dynamics of a counter-rotating four-vortex system was used as test case for four different LES codes employing different discretization schemes and subgrid-scale closure models [53]. The comparison indicated rather small differences in various features of the dynamics, such as the 
transfer of energy from 2D to 3D flow structures, the level of energy dissipation, the timeliness of the linking and reconnection processes. Another non-published DLR in-house benchmark of four different LES codes indicated that the results are very similar and robust in highly stratified environments whereas vortex decay in neutrally stratified turbulence revealed significant deviations. Interestingly, these deviations may even be exceeded when the identical code is applied to atmospheric turbulence of the same eddy dissipation rate but with varying integral turbulence length scales [54]. On one hand, the found good agreements between the codes may be considered a confidence-building result. On the other hand, the deviations between the different turbulence scenarios indicate that peculiarities of the environmental turbulence may have a dominant effect on wake vortex decay.

\subsection{Fast-time models}

Fast-time wake-vortex prediction models are being developed for operational applications. For example, proposed procedures can be evaluated using the evolution of wakes under various atmospheric conditions and various flight paths. Fast-time models are also core elements of the predictive systems for the optimization of aircraft separations introduced in the next section. The first fast-time model suggested by Greene in 1986 [55] served as inspiring example for subsequent model developments. Some of the fast-time models in use include APA [56], TDP [57], P2P [15] and DVM [58]. They are using physics-based empirical parameterizations to mimic vortex transport and decay. The models are generally normalized employing e.g. length and time scales based on initial vortex circulation and separation. Model components and constants are adjusted according to theoretical considerations, results of numerical simulations, and field experiments [59] and are validated against data of the latter. To consider stochastic wake vortex behaviour some models add probabilistic components accounting for the uncertainty and variability of the initial and environmental conditions in order to predict the bounds of wake vortex behaviour in a probabilistic sense [15]. Recently, different versions of the fast-time models APA, TDP and P2P have been combined to a multi-model ensemble providing enhanced deterministic and probabilistic prediction skills [60]. See also Section 3.10. for aircraft modelling issues.

\subsection{Predictive Systems}

To predict vortex behavior the ambient meteorology must be measured for short-term behavior and forecasted for long-term behavior. The meteorological data is combined with wake vortex models (see previous section) and wake hazard models to predict when the vortices are sufficiently diminished in strength or have advected out of the path of a following aircraft. Early studies focused on vortex motion, but the many campaigns devoted to vortex decay are now supplementing vortex motion with the vortex decay particularly when vortex motion alone cannot lead to reduced vortex separations. While ground-based wake vortex 
advisory systems aim at optimising aircraft separations for approach and landing or departures, airborne systems rather target wake turbulence awareness and avoidance of encounters.

NASA developed the Aircraft VOrtex Spacing System (AVOSS) to demonstrate the feasibility of providing weather-dependent wake-vortex spacing criteria for approach and landing by automated collection of relevant weather data, prediction of wake vortex behavior, and derivation of safe wake-vortex spacing criteria [61]. DLR's Wake Vortex Prediction and Monitoring System (WSVBS) supports dynamic adjustment of aircraft separations dependent on weather conditions and the resulting wake vortex behaviour [62],[63]. While the system was initially developed to tactically increase airport capacity for approach and landing on closelyspaced parallel runways, it was further developed for dynamic pair-wise separations considering aircraft type pairings and environmental conditions (corresponding to RECAT Phase 3, see section 2.11) [64].

While the airborne wake encounter prevention system (WEPS) developed in SESAR project 9.11 and DLR's Wake Encounter Avoidance and Advisory system (WEAA) are using aircraft and meteorological data transmitted by ADS-B-In in order to predict wake vortices generated by surrounding aircraft, the Integrated Wake Vortex Safety System (IWVSS) proposed by the Russian State Institute of Aviation Systems (GosNIIAS) predicts the wake of its own ship and transmits the data via ADS-B-Out. Successful flight tests have demonstrated the basic functionality of the airborne approach [65]. Recommendations for the integration of missing data into the ADS-B protocols have been devised [66],[67].

\subsection{Airborne Sensors}

Airborne wake sensors have been suggested for years and a few experimental systems have been developed [68]. A short pulse direct measuring UV lidar for the measurement of gusts, turbulence and wake vortices in front of the aircraft has been developed and flight-tested on the DLR ATTAS and the A340 aircraft [68]. The results suggest that relative wind velocities can be measured with a standard deviation of below $2 \mathrm{~m} / \mathrm{s}$ even at high altitudes with no appreciable aerosol concentrations. Such measurements in the near-field ahead of the aircraft can be used as input to wake impact alleviation control systems that may alleviate the aircraft's response during the wake encounter by counteracting control-surface deflections [69].

\subsection{Current Systems}

Based on extensive analysis of wake vortex behavior, new procedures and separation standards are being developed and implemented. Over time, it is expected that the new separation standards will be implemented worldwide. Eventually, wake turbulence separation may evolve into a dynamic and specific aircraft-to-aircraft relationship based on individual aircraft wake characteristics. 
However, there are three initiatives being implemented today: (a) Wake Turbulence Recategorization by the FAA in the US [70] and by EuroControl in Europe [71]; (b) Dependent Staggered Approaches to Closely Spaced Parallel Runways by the FAA in the US and (c) Wake Turbulence Mitigation for Departures by the FAA in the US.

In Wake Turbulence Recategorization, the traditional maximum-certificated- takeoff-weightbased separation standard is replaced with a separation matrix based on wake vortex physics and aircraft dynamics parameters, namely wingspan, maximum takeoff weight, final approach speed and roll moment capability [72]. The methodologies of the US and European Recategorization systems are similar but differ in some of the computational details. Pulsed lidars were used to record the decay of vortices for each aircraft type, nominally at one wingspan height as it is the worst-case region for a wake encounter. The longest lasting $5 \%$ of the vortices of each aircraft type was then used to determine the decay function for the worstcase decay (i.e., longest-lasting vortices). Selected airports in the US and Europe have been or are being implemented with the Phase I (six static categorical wake turbulence separations) system. The US also has implemented Phase II (static pairwise wake turbulence separations) systems.

Dependent Staggered Approaches to Closely Spaced Parallel Runways enables the use of dependent 1.5-nautical mile diagonal separation between two aircraft on closely spaced parallel runways (parallel runways less than 2500 feet (762 meters) apart) during instrument approaches with Medium and Light aircraft as leaders in the pairing sequence. Wake Turbulence Mitigation for Departure is the first automation-driven wake separation change that enables dynamic separation for departures on parallel runways separated by less than 2500 feet (762 meters) based on meteorology, namely the crosswind.

\subsection{Vortex Encounters}

As confirmed by the ASRS [73], the primary areas of concern are on approach and landing, climb through and descend through at altitude. On approach and landing there have been no accidents when both aircraft (the leading or vortex-generating aircraft and the following or vortex-encountering aircraft) fly the glideslope and are separated by the current separation standards. However, incidents in this flight phase are reported quite regularly at major airports [74],[75].

Flight tests and simulations of vortex encounters have been conducted. Fig. 3 delineates aircraft reactions at different positions within a wake-vortex flow field. Given sufficient altitude, the flight tests have shown that the best response is to let the aircraft fly out of the vortex system. Pilot-induced actions can aggravate the situation and have led to structural failures. Numerous 
simulations have been done, both in the 1980s (primarily by NASA [77]) and more recently ([78], [79] and FAA, for example).

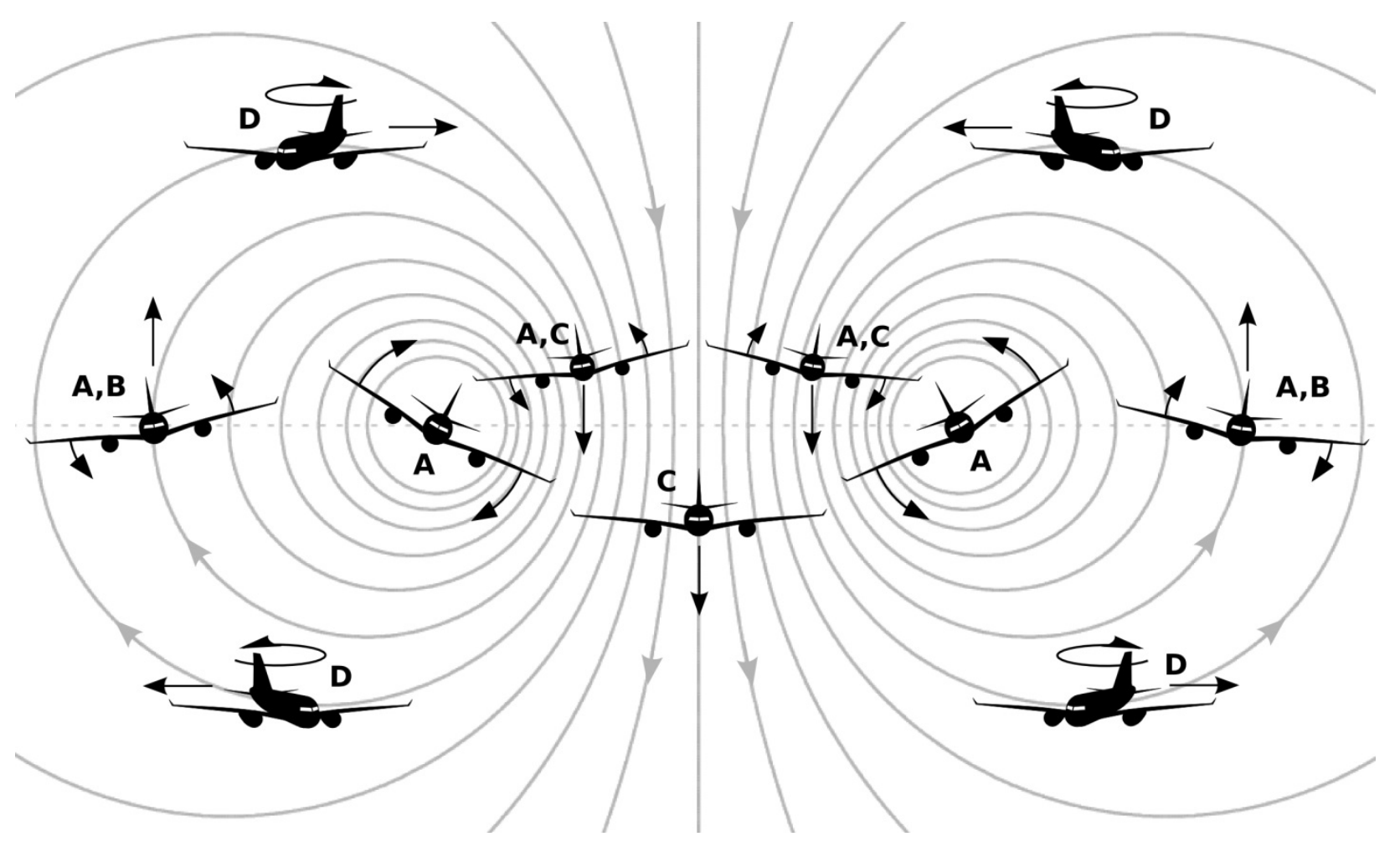

Fig.3. Aircraft reactions in wake-vortex flow field [13],[76]. (A) roll motion, (B) upward motion, (C) downward motion, (D) yaw motion.

Simulations have been used to examine the influence of vortex curvature on encounter severity ([80],[81],[82],[83],[84]). As noted by Crow [11], wakes deform as a result of the long-wave Crow instability, which in turn is driven by the ambient turbulence and in later stages by the temperature stratification [83]. With increasing turbulence intensity and increasing turbulence integral length scales the vortex topology is becoming more complex and the classical shape of the Crow instability and the ring formation is getting lost in favor of superimposed random deformations caused by large scale turbulent eddies [34]. Piloted-flight-simulator experiments conducted by Bienick and Luckner [82] indicate increased bank angle upsets during the onset of the Crow instability, but significantly lesser upsets during flight through vortex rings. On the other hand, auto-pilot simulations by Vechtel [84] demonstrate that the encounter response may decrease from straight vortices over wavy vortices to vortex rings. Only shortly prior to linking aircraft responses may be higher than with similar strength straight vortices, because the high velocities close to the approaching vortex cores may impact the aircraft simultaneously. 
Rotorcrafts share the airspace in the terminal environment with many fixed-wing aircraft. Limited flight tests have shown that a rotorcraft encountering a wake vortex is less severe than a comparably sized fixed-wing aircraft encountering the same vortex. Reference [24] describes flight tests where a lidar was used to measure the strength and decay of rotorcraft vortices. Very limited measurements have been made in a runway environment. The flow field trailing behind a rotorcraft is very complex; as the forward speed increases the flow field transitions from all downwash to a mixture of downwash and a trailing vortex pair to all trailing vortex pair. Typically, forward flight from 0 to about 10 knots is accompanied by just a downwash flow, forward flight from about 10 to about 35 knots is characterized initially (10 knots) by a downwash which transitions to zero at about 35 knots but is accompanied initially by no rolled up vortex pair at 10 knots and by only the vortex pair by about 35 knots. Above 35 knots the vortex pair behind a rotorcraft is very much like the vortex pair seen behind fixed-wing aircraft.

\section{Gaps in our Knowledge}

In this part of the paper, the sections above where the state of the current knowledge of wake vortex behavior is described are extended to discuss the known gaps in this knowledge. Obviously, these gaps reflect the opinions of the authors; other points of view are welcome and should be expressed so that all the important gaps are addressed in current and future projects.

\subsection{Long-Distance Vortex Transport}

The behavior of vortices transporting over long distances is an important consideration in the operation of parallel and intersecting runways. Many airports have parallel runways separated by less than the 2500 feet (762 meters) now required for operation as independent VFR runways when considering the wake-vortex hazard. Measurements to date have shown that the 2500-foot (762-meter) rule is very conservative, but this has not yet been confirmed with the new very heavy aircraft now flying in commercial operation. Based on past measurements as well as measurements of these new very heavy aircraft and the degree of hazard posed by these long-distance transport vortices, a vortex safe lateral separation could be established.

\subsection{Departure Vortices}

Measurement of departure vortices, especially near the ground, is more difficult due to their spread in flight paths compared to landing operations. The vortices are different as the aircraft are using less flaps and flying faster, but this does lead to having more roll control. Even with these differences, it has been shown that departure vortices have similar vortex strengths and decay rates when compared to landing vortices [85],[86]. 
The main concern is operational. A lead aircraft could climb faster than the following aircraft. Conditions may preclude the lead or follower aircraft from turning away from runway orientation.

\subsection{High-Altitude Vortex Behavior}

For many years, the FAA Wake Turbulence Advisory Circular and other guidance material indicated that aircraft vortices will descend at high altitudes as much as 900 feet. With the introduction of very Heavy and Super aircraft, in calm atmospheric conditions their vortices have been observed to descend 1000 feet (305 meters) and more. It is important to bound the descent distance as well as the attendant meteorological conditions and to determine the strength or circulation of the vortices that have descended 1000 feet (305 meters) or more.

At the high altitudes, severe vortex encounters can occur at large separation distances; a safe 5mile separation on approach maps into a 20-mile separation at en route speeds.

Serious encounters are also being reported for climb and descent through operations. Procedures such as the following aircraft moving upwind are currently being investigated.

\subsection{Quantitative Hazard Definition}

All safety analyses have used comparative measures, such as the new situation is "no worse than current ICAO separations" or "less than or equal to" some existing operation. As new aircraft designs or certain reduced aircraft separations are proposed it will become necessary to quantify the degree of hazard posed by the new entity. Is the degree of hazard a specific roll angle or roll moment or loss of altitude when two aircraft are subject to a new operation (i.e., closer lateral separation, perpendicular vortex encounters, reduced vertical separation, etc.)?

\subsection{Other Aircraft}

In the near future, there are many technologies that are expected to be introduced into commercial aviation. Wide-bodied aircraft are getting heavier and heavier; even rotorcraft are becoming heavier and heavier. Supersonic aircraft may return, especially if the aircraft can fly over land due to new means to lessen the sonic boom. Delta wing aircraft will introduce new issues as will unmanned commercial flights.

\subsection{Predictive Systems}

As indicated above, new systems are being implemented (RECAT and RECAT-EU) that decrease separations during approach and landing. Here, the familiar Heavy/Medium/Light categories are expanded to six categories, which permit certain aircraft pairs to operate safely with less 
separation and thus increase the airport throughput. These new systems are not predictive but reflect reduced separations for specific aircraft leader/follower pairs.

Predictive systems primarily depend on the ambient meteorology. A strong enough crosswind, for example, would clear an approach corridor of vortices and a leader/follower pair could proceed safely with less than current ICAO separations assuming runway occupancy and collision risk are not issues. Now the safety concern rests on the ability to predict when and how long an approach corridor is free of vortices and thus the ability to predict the meteorology is of prime importance. The questions now become when will the crosswinds permit decreased separations, what separation is possible, how long will the conditions be appropriate for using decreased separations, how far up the glide path must the approach corridor be free of vortices, will the time interval when decreased separations are safe be long enough to warrant using the decreased separations, can one forecast when appropriate conditions will be available, what is the procedure if the winds shift or decrease in magnitude (missed approach?), ...?

Although crosswinds for single runway approaches are described here, the situation is similar for single runway departures and for operations on closely-spaced parallel runways. Research is continuing on how to integrate the local meteorology with operational runways. Crosswind was used as the example, but are there other indicators that can be used (i.e., like turbulence or solar insolation).

\subsection{Airborne Sensors}

How can airborne sensors be designed to be robust and low-cost such that airlines would actually order them? Possibly, a multiple use system comprising short and long distance detection of not only wake vortices but other hazards such as turbulence, gusts, ice crystals, volcanic ash plus independent true airspeed measurement could be a good argument. Issues about the use of airborne systems need to be examined, such as increased workload during times of high workload, responses to warnings, too frequent warnings and appropriate thresholds and even their effectiveness for leading aircraft with weak axial flows in their vortices.

\subsection{Vortex Alleviation}

The measurements of wake vortex decay comparing approaching aircraft with and without winglets should be extended to phases of flight where the main vortices detach from the winglet tips and not from the flaps as during approach and landing.

The acceleration of wake vortex decay close to the ground via plate lines should be demonstrated in the environment of an international airport in order to quantify the real-life 
benefits of this method. For this purpose, a plate line design must be elaborated that is compatible with airport requirements (e.g. stability, frangibility) and may obtain approval by authorities.

\subsection{Computational Fluid Dynamics}

Although CFD of wake vortices appears to be quite mature, a number of challenges and opportunities for further development still persist. Despite the sustained increase of computing power the challenge of simultaneously resolving large atmospheric eddies (requiring large meshes) and the preservation of tight vortex cores (requiring high spatial resolution) persists. This dilemma can be partly overcome by employing subgrid-scale models that meet the complex interaction of turbulence and rotation at high Re-numbers.

Another aspect is the consideration of specific aircraft types, aircraft configurations, and flight phases, which requires modeling the flow around the aircraft and the subsequent vortex roll-up to the counter-rotating vortex pair. A CFD method providing a reliable aircraft specific footprint on its wake would constitute an excellent tool for the efficient development and assessment of aircraft designs with weaker or quicker disintegrating vortices. In addition, the role of aircraft induced turbulence stemming from high-lift devices, landing gear but most importantly from the fuselage wake and the engine jets may have significant impact on wake vortex decay characteristics [14].

A long-term vision may be the virtual flight in realistic environments where a specific aircraft type may fly through realistic gusts or turbulence in LES quality and the impact on the aircraft is considered [87]. At the same time, the wake vortices rolling up from that flow may be influenced by both the oncoming flow characteristics and the aircraft reactions and maneuvers. Other applications may comprise CFD simulations of wake vortex encounters including the aircraft reaction, its structural response, and potential flow detachment on the wings or formation flight.

So far, CFD mainly furthered the understanding of the physics of wake vortices and their interaction with the environment. Any new developments of procedures and separation standards mainly rested upon lidar measurements. In order to increase confidence of safety regulators in CFD (or fast-time models if statistics are required) it would be necessary to further close the gap between measurements and simulations systematically. That is the capability to reproduce lidar measurements in sufficient accuracy must be demonstrated in a convincing way. This could be achieved for example by the consideration of key data used within RECAT or by the reproduction of a set of scenarios defined by experts. On that way it may be helpful to simulate lidar measurements employing LES data [88] in order to better understand the peculiarities of specific lidar setups and processing algorithms. Once sufficient confidence in 
CFD or fast-time models has been established, these methods could potentially replace parts of the expensive lidar data gathering campaigns.

\subsection{Fast-time models}

As noted in Section 2.8, most of the fast-time models incorporate parameterizations considering specific behavior in certain meteorological or geometrical conditions, such as faster decay near the ground. What is missing in the wake behavior models is better modeling of the wing loading. Most models assume elliptic wing loading both for its convenience and because the initial separation of vortices for many aircraft are close to elliptical. However, new aircraft wing designs are likely to deviate from current practice. Initial vortex separation, $b_{0}=s B$ is characterized by the spanwise load factor, $s$, which amounts to $\pi / 4$ for elliptic wing loading and may deviate from that depending on wing design and aircraft configuration. The initial circulation, $\Gamma_{0}=M g / \rho s B V$ is only inversely proportional to $s$, where $M g$ denotes the aircraft weight, $\rho$ the air density, $B$ the wing span, and $V$ the airspeed. However, the vortex time scale, $t_{0}=2 \pi b_{0}^{2} / \Gamma_{0}$ and thus the vortex lifetime scales with $s^{3}$ and vortex descent speed, $w_{0}=b_{0} / t_{0}$ with $s^{-2}$. Effects of stable thermal stratification scale with $s^{3}$ (normalized Brunt-Väisälä frequency, $\left.N t_{0}\right)$ and turbulence dissipation rate with $s^{7 / 3}\left(\left(\varepsilon b_{0}\right)^{1 / 3} / w_{0}\right)$. These dependencies stress the high relevance of the consideration of initial vortex spacing on the prediction skills of fast-time models. Regardless, onboard tools may be developed to support concepts such as dynamic separation of aircraft at all aircraft locations (takeoff, climb, cruise, descent, holding, approach and landing). Thus, flap positions on the wing and the amount of deployment will be important and need to be incorporated into detailed wake behavior models.

\section{Summary}

This review has shown that much has been learned about aircraft wake vortices. The introduction of Heavy aircraft, particularly the B747-100 in 1969, led to aircraft wake separation standards and the beginning of extensive studies of wake characteristics. Initially, sensors and sensor systems were developed to measure wake behaviour in the terminal environment. Meteorological sensors were then introduced to correlate the weather with wake vortex behaviour. The extensive data and CFD were used to develop wake models, which are now used to evaluate potential new procedures. These tools are now being used to mitigate the impact of wake vortices on terminal operations with systems such as RECAT.

However, there is much more to be learned. More sophisticated CFD and meteorological measurements will facilitate better forecasting of conditions when wake vortex separations can be decreased and thus gain more airport throughput for the ever increasing air traffic operations worldwide. 


\section{Glossary}

\begin{tabular}{|c|c|}
\hline ADS-B & Automatic Dependent Surveillance - Broadcast \\
\hline AIAA & American Institute of Aeronautics and Astronautics \\
\hline APA & Aircraft vortex spacing system Prediction Algorithm \\
\hline ASRS & Aviation Safety Reporting System \\
\hline ATM & Air Traffic Management \\
\hline AVOSS & Aircraft Vortex Spacing System \\
\hline CFD & Computational Fluid Dynamics \\
\hline DOT & Department of Transportation \\
\hline DLR & Deutsches Zentrum für Luft- und Raumfahrt e.V., German Aerospace Center \\
\hline DVM & Deterministic wake Vortex Model \\
\hline EU & European Union \\
\hline FAA & Federal Aviation Administration \\
\hline GosNIIAS & Russian State Institute of Aviation System \\
\hline ICAO & International Civil Aviation Organization \\
\hline IWVSS & Integrated Wake Vortex Safety System \\
\hline LES & Large Eddy Simulation \\
\hline MA & Massachusetts \\
\hline NASA & National Aeronautics and Space Administration \\
\hline P2P & Probabilistic 2-Phase wake vortex model \\
\hline $\operatorname{Re}$ & Reynolds number \\
\hline RECAT & Recategorization \\
\hline$R \& D$ & Research and Development \\
\hline SESAR & Single European Sky ATM Research \\
\hline TDP & Terminal area simulation system Derived algorithms for wake Prediction \\
\hline TU & Technical University \\
\hline US & United States \\
\hline VFR & Visual Flight Rules \\
\hline WEAA & Wake Encounter Avoidance and Advisory system \\
\hline WEPS & Wake Encounter Prevention System \\
\hline $2 / 3 D$ & 2 or 3 Dimensions \\
\hline$b_{0}$ & initial vortex separation \\
\hline$S$ & spanwise load factor \\
\hline$t_{0}$ & vortex time scale \\
\hline$w_{0}$ & descent rate \\
\hline$B$ & wing span \\
\hline$M g$ & aircraft weight \\
\hline$N$ & Brunt-Väisälä frequency \\
\hline
\end{tabular}


airspeed

$\Gamma_{0} \quad$ initial circulation

$P \quad$ air density

\section{References}

[1] Hallock JN. Aircraft Wake Vortices: An Assessment of the Current Situation. Report No. DOTFAA-RD-90-29, January 1991, 59pp.

[2] Widnall SE. The structure and dynamics of vortex filaments. Annu Rev Fluid Mech 1975;7:141-165.

[3] Donaldson CduP, Bilanin AJ. Vortex Wakes of Conventional Aircraft. AGARD-AG-204, May 1975, 79 pp.

[4] Hallock JN, Eberle WR, editors. Aircraft Wake Vortices: A State-of-the-Art Review of the United States R\&D Program. Report FAA-RD-77-23, February 1977, 346pp.

[5] Hallock JN, Greene GC, Burnham DC. Wake Vortex Research - A Retrospective Look. Air Traf Contr Quart 1998;6(3):161-178.

[6] Spalart PR. Airplane Trailing Vortices. Annu Rev Fluid Mech 1998;30: 107-138.

[7] Rossow VJ. Lift-generated vortex wakes of subsonic transport aircraft. Prog Aerosp Sci 1999;35(6):507-660.

[8] Gerz T, Holzäpfel F, Darracq D. Commercial Aircraft Wake Vortices. Prog Aerosp Sci 2002;38(3):181-208.

[9] Elsenaar B et al. Wake Vortex Research Needs for "Improved Wake Vortex Separation Ruling" and "Reduced Wake Signatures". Part II, Specialist's Reports, Final Report of the Thematic Network "WakeNet2-Europe", 6th Framework Programme, 2006, 114pp.

[10] Aircraft Wake Vortex State-of-the-Art \& Research Needs. WakeNet3-Europe, compiled by F. Holzäpfel et al., issued by A. Reinke, C. Schwarz, November 2015, 201 pages.

[11] Breitsamter C. Wake vortex characteristics of transport aircraft. Prog Aerosp Sci 2011;47:89-134.

[12] Stephan A, Holzäpfel F, Misaka T. Hybrid simulation of wake-vortex evolution during landing on flat terrain and with plate line. Int J Heat Fluid FI 2014;49:18-27.

[13] Körner S. Multi-Model Ensemble Wake Vortex Prediction. Doctoral thesis, Deutsches Zentrum für Luft- und Raumfahrt, Institut für Physik der Atmosphäre, Dissertation an der Rheinisch-Westfälischen Technischen Hochschule Aachen, Forschungsbericht 2017-44, 2017.

[14] Misaka T, Holzäpfel F, Gerz T. Large-Eddy Simulation of Aircraft Wake Evolution from Roll-Up until Vortex Decay. AIAA J 2015;53(9):2646-2670. 
[15] Holzäpfel F. Probabilistic Two-Phase Wake-Vortex Decay and Transport Model. J Aircraft 2003;40(2):323-331.

[16] Garodz L, Lawrence DM, Miller NL. Measurement of the Trailing Vortex Systems of Large Transport Aircraft Using Tower Fly-By and Flow Visualization (Summary, Comparison and Application). Report No. FAA-RD-75-127, May 1975.

[17] Burnham DC, Hallock JN, Kodis RD, Sullivan TE. Vortex Sensing Tests at NAFEC. Report No. DOT-TSC-FAA-72-2, January 1972, 72pp.

[18] Misaka T, Obayashi S, Stephan A, Holzäpfel F, Gerz T, Nakahashi K. Numerical Simulation of Jet-Wake Vortex Interaction. AIAA-2014-0926, 2014. 9pp.

[19] Burnham DC, Hallock JN. Motion of Aircraft Wake Vortices in Ground Effect. Report DOT-FAA-AAR-00-16, April 2000, 102.

[20] Hallock JN, Osgood SP, Konopka J. Wake Vortex Effects on Parallel Runway Operations. AIAA Paper No. 2003-0379, January 2003. 11pp.

[21] Hallock JN, Wang FY. Summary Results from Long-Term Wake Turbulence Measurements at San Francisco International Airport, Project Memorandum DOT-VNTSCFA27-PM-04-13, July 2004.

[22] Lissaman PBS, Crow SC, MacCready Jr PB, Tomback IH, Bate Jr ER. Aircraft Vortex Wake Descent and Decay under Real Atmospheric Effects. Report FAA-RD-73-120, October 1973, $222 \mathrm{pp}$.

[23] Holzäpfel F. Effects of Environmental and Aircraft Parameters on Wake Vortex Behavior. J Aircraft 2014;51(5): 1490-1500.

[24] Teager SA, Biehl KJ, Garodz LJ, Tymczyszym JJ, Burnham DC. Flight Test Investigation of Rotorcraft Wake Vortices in Forward Flight, Report DOT/FAA/CT-94/117, February 1996, 94pp.

[25] Crow SC. Stability Theory for a Pair of Trailing Vortices. AIAA J 1970;8(12):2171-2179.

[26] Burnham DC, Hallock JN. Decay Characteristics of Wake Vortices from Jet Transport Aircraft. J Aircraft 2013;50(1):82-87.

[27] Holzäpfel F, Gerz T, Baumann R. The turbulent decay of trailing vortex pairs in stably stratified environments. Aerosp Sci Technol 2001;5:95-108.

[28] Corjon A, Risso F, Stoessel A, Poinsot T. Three-Dimensional Direct Numerical Simulations of Wake Vortices: Atmospheric Turbulence Effects and Rebound with Crosswind. In: The characterization and modification of wakes from lifting vehicles in fluids, AGARD CP 584, May 1996. p. 28.1-21.

[29] Holzäpfel F, Hofbauer T, Darracq D, Moet H, Garnier F, Ferreira Gago C. Analysis of wake vortex decay mechanisms in the atmosphere, Aerosp Sci Technol 2003;4: 263-275.

[30] Pruis MJ, Delisi DP, Jacob D, Lai DY. Summary of NASA Wake and Weather Data Collection at Memphis International Airport: 2013-2015. AIAA Paper 2016-3274, June 2016, $12 \mathrm{pp}$. 
[31] Köpp F, Rahm S, Smakikho I, Dolfi A, Cariou J-P, Harris M. Comparison of Wake-Vortex Parameters Measured by Pulsed and Continuous-Wave Lidars. J. Aircraft 2005;42(4): 916923.

[32] Hallock JN. Monitoring Wake Vortex Strength near the Ground. J. Aircraft 1976;13(10):830-832.

[33] Proctor FH, Ahmad NN, Switzer GS, Limon Duparcmeur FM. Three-Phased Wake Vortex Decay, AIAA 2010-7991, 2010. 11 pp.

[34] Holzäpfel F, Misaka T, Hennemann I. Wake-Vortex Topology, Circulation, and Turbulent Exchange Processes. AIAA Paper 2010-7992, August 2010, 16pp.

[35] Chen S, Soares M, Wang, F Y. A Case Study on Aircraft Wake Vortex Evolution With and Without Winglets, In Preparation.

[36] Holzäpfel F, Stephan A, Heel T, Körner S. Enhanced Wake Vortex Decay in Ground Proximity Triggered by Plate Lines, Aircr Eng Aerosp Tec 2016;88(2):206-214.

[37] Takami H. A Numerical Experiment with Discrete Vortex Approximation with Reference to the Rolling Up of a Vortex Sheet. Stanford University, 1964.

[38] Hill FM. A numerical study of the descent of a vortex pair in a stably stratified atmosphere. J Fluid Mech 1975;71:1-13.

[39] Burnham DC, Hallock JN, Tombach IH, Brashears MR, Barber MR. Ground-Based Measurements of a B-747 Aircraft in Various Configurations. Report No. FAA-RD-78-146, December 1978.

[40] Hecht AM, Bilanin AJ, Hirsh JE. Turbulent Trailing Vortices in Stratified Fluids. AIAA J 1981;19:691-698.

[41] Schilling V, Siano S, Etling D. Dispersion of aircraft emissions due to wake vortices in stratified shear flows: A two-dimensional numerical study. J Geophys Res 1996;101:2096520974.

[42] Robins RE, Delisi DP. 3-D Calculations Showing the Effects of Stratification on the Evolution of Trailing Vortices. Note Num FI 1996;49:264-270.

[43] The Characterisation \& Modification of Wakes from Lifting Vehicles in Fluids. AGARD CP 584, May 1996.

[44] Spalart PR, Wray AA. Initiation of the Crow instability by atmospheric turbulence. In: The characterization and modification of wakes from lifting vehicles in fluids, AGARD CP 584, May 1996. p. 18.1-8.

[45] Gerz T, Ehret T. Wake dynamics and exhaust distribution behind cruising aircraft. In: The characterization and modification of wakes from lifting vehicles in fluids, AGARD CP 584, May 1996. p. 35.1-12.

[46] Switzer GF, Proctor FH. Numerical Study of Wake Vortex Behavior in Turbulent Domains with Ambient Stratification. AIAA 2000-0755, 2000. 14 pp. 
[47] De Visscher I, Bricteux L, Winckelmans G. Aircraft Vortices in Stably Stratified and Weakly Turbulent Atmospheres: Simulation and Modeling. J. Aircraft 2013;51(3):551-566.

[48] Misaka T, Holzäpfel F, Gerz T, Manhart M, Schwertfirm F. Vortex bursting, tracer transport, and decay mechanisms of a counter-rotating vortex pair. Phys Fluids 2012;24(2):25104.1-21.

[49] Lewellen DC, Lewellen WS. The Effects of Aircraft Wake Dynamics on Contrail Development. J Atmos Sci 2001;58:390-406.

[50] Stumpf E. Study of Four-Vortex Aircraft Wakes and Layout of Corresponding Aircraft Configurations. J Aircraft 2005;42(3):722-730.

[51] Holzäpfel F, Hofbauer T, Gerz T, Schumann U. Aircraft Wake Vortex Evolution and Decay in Idealized and Real Environments: Methodologies, Benefits and Limitations. In: Friedrich R, Rodi W, editors. Fluid Mec A 2002;65, Advances in LES of Complex Flows. Kluwer Academic Publishers. 2002. p. 293-309.

[52] Proctor FH. Numerical Study of a Long-Lived, Isolated Wake Vortex in Ground Effect. AIAA 2014-2469, 2014. 11 pp.

[53] Dufresne L, Baumann R, Gerz T, Winckelmans G, Moet H, Capart R, Cocle R, Nybelen L. Large-Eddy Simulation of Wake Vortex Flows at Very High Reynolds Numbers: A Comparison of Different Methodologies. Report No.D1.1.4-16, AWIATOR, August 2005, 39pp.

[54] Hennemann I, Holzäpfel F. Large-eddy simulation of aircraft wake vortex deformation and topology. Proc. IMechE Part G: J. Aerosp Eng 2011;225(12):1336-1350.

[55] Greene GC. An Approximate Model of Vortex Decay in the Atmosphere. J Aircraft 1986;23(7):566-573.

[56] Proctor FH. The NASA-Langley Wake Vortex Modelling Effort in Support of an Operational Aircraft Spacing System. AIAA Paper 1998-0589, January 1998.

[57] Proctor F, Hamilton DW, Switzer GF. TASS Driven Algorithms for Wake Prediction. AIAA Paper 2006-1073, January 2006.

[58] De Visscher IG, Winckelmans G, Lonfils T, Bricteux L, Duponcheel M, Bourgeois N. The WAKE-4D Simulation Platform for Predicting Aircraft Wake Vortex Transport and Decay: Description and Examples of Application. AIAA Paper 2010-7994, August 2010.

[59] Proctor FH, Hamilton DW, Han J. Wake Vortex Transport and Decay in Ground Effect: Vortex Linking with the Ground. AIAA Paper 2000-0757, January 2000.

[60] Körner S, Ahmad NN, Holzäpfel F, Van Valkenburg RL. Multimodel Ensemble Methods for Prediction of Wake-Vortex Transport and Decay. J Aircraft 2017;54(5):1849-1859.

[61] Hinton DA. Description of Selected Algorithms and Implementation Details of a ConceptDemonstration Aircraft VOrtex Spacing System (AVOSS). NASA TM-211027, 2001. 70pp.

[62] Holzäpfel F, Gerz T, Frech M, Tafferner A, Köpp F, Smalikho I, Rahm S, Hahn K-U, Schwarz C. The Wake Vortex Prediction and Monitoring System WSVBS - Part I: Design. Air Traf Contr Quart 2009;17(4):301-322. 
[63] Gerz T, Holzäpfel F, Gerling W, Scharnweber A, Frech M, Kober K, Dengler K, Rahm S. The Wake Vortex Prediction and Monitoring System WSVBS Part II: Performance and ATC Integration at Frankfurt Airport. Air Traf Contr Quart 2009;17(4):323-346.

[64] Holzäpfel F, Dengler K, Gerz T, Schwarz C. Prediction of Dynamic Pairwise Wake Vortex Separations for Approach and Landing, AIAA 2011-3037, 2011, 15 pp.

[65] Sölch I, Holzäpfel F, Abdelmoula F, Vechtel D. Performance of on-board wake vortex prediction systems employing various meteorological data sources, J Aircraft 2016;53(5):1505-1516.

[66] Standards Development Activities for using Near Real-Time Aircraft-Derived Data in Future Applications. RTCA DO-360, 2015, 68 pp.

[67] Aircraft Derived Meteorological Data via Data Link for Wake Vortex, Air Traffic Management and Weather Applications. Operational Services and Environmental Definition (OSED). RTCA DO-339, 2012, 98 pp.

[68] Schmitt NP, Rehm W, Pistner T, Zeller P, Diehl H, Navé P. The AWIATOR airborne LIDAR turbulence sensor. Aerosp Sci Technol 2007; 11:546-552.

[69] Ehlers J, Fischenberg D, Niedermeier D. Wake Impact Alleviation Control Based on Wake Identification. J Aircraft 2015;52(6):2077-2089.

[70] Cheng J, Tittsworth J, Gallo W, Awwad A. The Development of Wake Turbulence Recategorization in the United States. AIAA Paper 2016-3434, June 2016, 12pp.

[71] RECAT-EU European Wake Turbulence Categorisation and Separation Minima on Approach and Departure, EUROCONTROL, 2015, 32 pp.

[72] Hallock JN, Greene GC, Tittsworth JA, Strande PD, Wang FY. Use of Simple Models to Determine Wake Vortex Categories for New Aircraft. AIAA Paper 2015-3172, June 2015. $11 \mathrm{pp}$.

[73] Aviation Safety Reporting System, https://asrs.arc.nasa.gov/index.html.

[74] Critchley J, Foot P. UK CAA Wake Vortex Database: Analysis of Incidents Reported Between 1982 and 1990. Civil Aviation Authority, CAA Paper 91, 1991.

[75] Holzäpfel F. Analysis of potential wake vortex encounters at a major European airport. Aircr Eng Aerosp Tec 2017;89(5): 634-643.

[76] Luckner R, Höhne G, Fuhrmann M. Hazard criteria for wake vortex encounters during approach. Aerosp Sci Technol 2004; 8(8):673-687.

[77] Rossow VJ, Tinling BE. Research on Aircraft/Vortex-Wake Interactions to Determine Acceptable Level of Wake Intensity. J Aircraft 1988;25(6):481-492.

[78] Schwarz C, Hahn K-U. Full-flight simulator study for wake vortex hazard area investigation. Aerosp Sci Technol 2006;10(2): 136-143.

[79] Luckner R. Modeling and Simulation of Wake Vortex Encounters: State-of-the-Art and Challenges. AIAA Paper 2012-4633, August 2012. 
[80] Loucel RE, Crouch JD. Flight-Simulator Study of Airplane Encounters with Perturbed Trailing Vortices. J Aircraft 2005;42(4):924-931.

[81] Vechtel D. Flight Simulator Study on the Influence of Vortex Curvature on Wake Encounter Hazard Using LES Wind Fields. Aeronautical J 2012;111(1177):287-302.

[82] Bieniek D, Luckner R. Simulation of Aircraft Encounters with Perturbed Vortices Considering Unsteady Aerodynamic Effects. J Aircraft 2014;51(3):705-718.

[83] Bieniek D, Luckner R, De Visscher I, Winckelmans G. Simulation Methods for Aircraft Encounters with Deformed Wake Vortices. J Aircraft 2016;53(6):1581-1596.

[84] Vechtel D. Simulation study of wake encounters with straight and deformed vortices. Aeronautical J 2016;120(1226):651-674.

[85] Hallock JN. Differences between Landing and Takeoff Wake Vortices. Proceedings of the International Wake Vortex Meeting, Report TP 13166, December 1997, pp. 81-82.

[86] Mackey SM, Wang FY, Wassaf H, Soares M. Comparison between Arrival and Departure Wake Vortex Statistics near the Ground. 1st CEAS European Air and Space Conference, Report CEAS-2007-002, September 2007, pp. 1049-1054.

[87] Stephan A, Zholtovski S, Holzäpfel F. The effect of gusts on aircraft wake vortices. Aircr Eng Aerosp Tec 2017;89(5):692-702.

[88] Holzäpfel F, Gerz T, Köpp F, Stumpf E, Harris M, Young RI, Dolfi-Bouteyre A. Strategies for Circulation Evaluation of Aircraft Wake Vortices Measured by Lidar, J Atmos Ocean Tech 2003;20(8):1183-1195. 